\title{
REZENSIONEN
}

\section{Winklers Alterswerk: ein Meisterstück zur Geschichte der Zeit seit 1945}

\author{
Winkler, Heinrich August: Geschichte des Westens. Vom Kalten Krieg zum Mauerfall, Verlag \\ C.H. Beck, München 2014,1258 Seiten, € 39,95.
}

Winkler, Heinrich August: Geschichte des Westens. Die Zeit der Gegenwart, Verlag C.H. Beck, München 2015, 687 Seiten, $€ 29,95$.

Heinrich August Winkler, Jahrgang 1938, emeritierter Professor der Geschichtswissenschaft an der Humboldt-Universität zu Berlin, ist die personifizierte Produktivität. Nach seiner Promotion 1963 bei Hans Rothfels (wie sein Vater 1931), vielen gelehrten Studien, zunächst über die Weimarer Republik ${ }^{1}$, dann über die sperrige deutsche Geschichte, den „langen Weg nach Westen “2, erweiterte sich sein Forschungsfeld immer mehr; in gewisser Weise ist nun eine Art Weltgeschichte entstanden: 2009 erschien der erste Band der „Geschichte des Westens" von den Anfängen in der Antike bis zum 20. Jahrhundert, 2011 der nächste, die Zeit von 1914 bis 1945 umfassend, den „Zweiten Dreißigjährigen Krieg“.3 Im dritten Band fängt Heinrich August Winkler die turbulente Zeit „vom Kalten Krieg bis zum Mauerfall“ ein (Herbst 2014), im vierten die „Zeit der Gegenwart“ (Anfang 2015). Der Begriff des „Westens“ wird nicht in erster Linie geographisch gefasst, sondern im Sinne der Akzeptanz unveräußerlicher Menschenrechte, der Rechtsstaatlichkeit, Gewaltenteilung, Volkssouveränität und repräsentativen Demokratie. Insofern ist das Werk für die Politikwissenschaft gut anschlussfähig.

Der dritte Band, gegliedert in fünf große, chronologisch geordnete Kapitel, beginnt mit den Anfängen des Kalten Krieges (1945 bis 1949) und hört mit dem Abschied vom Kalten Krieg auf (1985 bis 1989). „Nie zuvor hatte der transatlantische Westen so sehr eine Einheit gebildet wie in den viereinhalb Jahrzehnten zwischen 1945 und 1990" (S. 17). Die erste Zäsur steht für den Sieg über den Nationalsozialismus, die zweite für den Sieg über den Kommunismus. Dazwischen sind die Kapitel „Vom Koreakrieg zur Kubakrise (1949 bis 1963), „Von der Konfrontation zur Entspannung“ (1963 bis 1975) und „Von der Entspannung zur Konfrontation“ (1975 bis 1985) angesiedelt. Im Vordergrund stehen die Ent-

1 Vgl. Heinrich August Winkler, Mittelstand, Demokratie und Nationalsozialismus. Die politische Entwicklung von Handwerk und Kleinhandel in der Weimarer Republik, Köln 1972; ders., Arbeiter und Arbeiterbewegung in der Weimarer Republik, drei Bände, Berlin / Bonn 1984-1987; ders., Weimar 1918-1933. Die Geschichte der ersten deutschen Demokratie, München 1993.

2 Vgl. ders., Der lange Weg nach Westen, Bd. 1: Vom Ende des Alten Reiches bis zum Untergag der Weimarer Republik; Bd. 2: Vom „Dritten Reich“ bis zur Wiedervereinigung, Paperback, München 2014 (Erstdruck jeweils 2000).

3 Vgl. ders., Geschichte des Westens. Von den Anfängen in der Antike bis zum 20. Jahrhundert, München 2009; ders., Geschichte des Westens. Die Zeit der Weltkriege 1914-1945, München 2011. 
wicklung in den wichtigsten Staaten (neben den USA die Sowjetunion, Deutschland, Frankreich, Großbritannien und Italien), die Konfliktherde auf der Welt sowie der Wettkampf der Systeme, in dem die Sowjetunion nach der Helsinki-Schlussakte 1975 - diese schrieb die Unverletzlichkeit aller Grenzen ebenso fest wie das Selbstbestimmungsrecht der Völker - immer mehr in die Defensive geriet - politisch wie ökonomisch. Der Leser erfährt viel Erhellendes, etwa zur „transnationalen Revolte“ der 68er (S. 582 - 626), die in den einzelnen Ländern aufgrund unterschiedlicher Anlässe spezifische Prägungen erfuhr. Ihr wird zugebilligt, die westlichen Gesellschaften in der Konsequenz liberalisiert zu haben, auch wenn die ursprünglichen Ziele verpufften. Die $68 \mathrm{er}$ veränderten die westlichen Gesellschaften, diese die 68er. Das 20. Jahrhundert gilt für Winkler mit Akira Iriye als das „amerikanische Jahrhundert“ (S. 18). Die Politik der USA steht daher im Vordergrund. Dabei idealisiert Winkler sie keineswegs, misst sie vielmehr an den Prinzipien des Westens (unveräußerliche Menschenrechte, Rechtsstaatlichkeit, Pluralismus), zeigt die Diskrepanz zwischen Theorie und Praxis auf. Im Kampf gegen den Kommunismus bedienten sich die USA oft zwielichtiger Verbündeter, etwa in Mittel- und Lateinamerika. Westliche Selbstkritik nahm daran Anstoß. Das ist alles richtig, aber zur ganzen Wahrheit gehört der Hinweis, dass der Westen den Kommunismus dann nicht kritisierte oder gar bekämpfte, wenn ein gemeinsamer Feind wie der Nationalsozialismus Geschlossenheit gebot.

Die Kehrseite der chronologischen Struktur: Kulturelle, militärische, ökonomische und soziale Faktoren kommen im Vergleich zur schieren Menge der politischen zu kurz. Zusammenhängendes wird unvermeidlich auseinandergerissen. Das gilt ebenso für die ersten beiden Werke wie für den Schlussband. Schließlich deckt sich die Grobgliederung nicht mit den Zäsuren in den jeweiligen Staaten, wie beispielsweise die Geschichte der Bundesrepublik Deutschland mit ihren großen Regierungswechseln 1969 und 1982 erhellt.

Der Leser ahnt, welche Menge an Quellen und Literatur der Autor gesichtet hat, wobei der Anmerkungsapparat erfreulich knapp ausfällt (S. 1143 - 1208). Sehr darauf bedacht, möglichst viele Ereignisse einzubeziehen, verliert Winkler, dessen Urteilskraft besticht, zuweilen den roten Faden aus dem Auge, und Faktenhuberei grassiert dann. Ein keineswegs gesuchtes Beispiel: „Von Anfang 1975 bis Juni 1976 fiel das britische Pfund von 2,40 auf 1,70 Dollar. Die Inflationsrate lag 1975 bei 24,2 und 1986 bei 15,9 Prozent. Die Bank of England hob im Juni 1976 den Diskontsatz auf 11,5 Prozent an. Eine auf sechs Monate befristete internationale Anleihe über 5,3 Milliarden Pfund zeitigte, teilweise bedingt durch Ernteausfälle auf Grund extremer Dürre, nur geringe Wirkungen. Im Oktober 1986 sank der Pfundkurs auf 1,55 Dollar; die Zahl der Arbeitslosen bis zum Jahresende auf 1,3 Millionen“ (S. 764). Unter dieser Faktenfülle leidet die Übersichtlichkeit, und die Verbindung zum normativen Projekt des Westens, Fluchtpunkt von Winklers Überlegungen, wirkt vielleicht etwas aufgesetzt. Aber das sind Petitessen.

Winkler hält in seinem Meisterwerk die Stalin-Note vom 10. März 1952 für eine Finte, nicht für eine Chance, die Wiedervereinigung auf demokratischer Basis zu erreichen. Ein neutrales Deutschland im Herzen Europas sei angesichts der geostrategischen Folgen (Rückzug der USA) nicht wünschenswert gewesen. „Nimmt man die inneren und die äußeren Wirkungen zusammen, die eine Wiedervereinigung auf der Basis der sowjetischen Vorschläge vom Frühjahr 1952 wahrscheinlich gehabt hätte, fällt die Behauptung von der ,versäumten Chance' jenes Jahres in sich zusammen" (S. 171). Er ruft mit großer Anschaulichkeit noch einmal die Zeit vor über 25 Jahren in Erinnerung: die Demonstrationen in Leipzig, die mit dem 9. Oktober 1989 die Freiheit erzwangen, und den Fall der Mauer, der 
die Einheit beschleunigte. Michail Gorbatschow spielte anders als Josef Stalin nicht die „deutsche Karte“. Die Verzahnung der komplexen, sich überschlagenden und nicht vorhersehbaren Ereignisse ist geglückt. Selbst der Chronist fällt der Geschwindigkeit des Wandels zum Opfer. So stimmten die „neuen“ Kräfte mit den „alten“ am Zentralen Runden Tisch in Berlin, am 7. Dezember 1989 ins Leben gerufen, keineswegs darin überein, die Eigenstaatlichkeit der DDR zu wahren. Schließlich hatten die Blockparteien der SED schnell die Loyalität aufgekündigt und sich bald zum Sprachrohr der auf eine baldige Einheit setzenden Bevölkerungsmehrheit gemacht, weswegen die erste und letzte demokratische Volkskammerwahl am 18. März 1990 mit einem überwältigenden Wahlsieg der unter der Führung der CDU stehenden „Allianz für Deutschland“ endete.

Für Winkler ist der Sowjetkommunismus ohne Wenn und Aber gescheitert. Seine These, er sei wegen des Gleichheitsgedankens dem Westen näher verbunden als der Nationalsozialismus, ist insofern problematisch, als das kommunistische Gleichheitsprinzip sich weder in der Theorie noch in der Praxis mit dem westlichen deckt. Und wie verhält sich die These zu seiner anderen, wonach Stalin, „wenn man auf die Gleichschaltung der Gesellschaft mit den Mitteln von Propaganda und Terror abstellt, ein noch totalitäreres Regime, als es das Hitlers in Deutschland gewesen war" (S. 174 f.), errichtet hat? Gorbatschow kommt wohl zu gut weg, weil die Überwindung des totalitären Systems zwar die Folge seiner Politik war, nicht jedoch die Intention. Zu Recht gilt die Zeit nach Ende der 1980er Jahre als „Welt ohne Gleichgewicht“ (S. 1117). Winkler liegt näher bei Samuel P. Huntington, der einen „Kampf der Kulturen“ heraufziehen sah und dabei allerdings „die subversive Kraft des normativen Projekts des Westens“ (S. 1123) unterschätzt habe, als bei Francis Fukuyama mit seiner These vom „Ende der Geschichte“.

Der letzte, ursprünglich nicht vorgesehene Band behandelt die „Zeit der Gegenwart“, also die Periode, die bekanntlich noch qualmt, um mit Barbara Tuchman ${ }^{4}$ zu reden: Zur Sprache kommen unter anderem die Beendigung des Bosnienkonfliktes, das Russland Boris Jelzins, der Zusammenbruch des italienischen Parteiensystems, die Vertiefung der Europäischen Union, die Weltfinanzkrise, der „arabische Frühling“, der „Islamische Staat“. Das alles einzuordnen stellt eine besondere Herausforderung dar, da vieles im Fluss ist. Die Gliederung mit den Großkapiteln „Vom Triumph zur Tragödie: 1991-2001“, „Vom „Krieg gegen den Terror' zur Weltfinanzkrise: 2001-2008“ und „Das Ende aller Sicherheit: 20082014“ leuchtet ein. Die letzte Überschrift, die auf die „fortschreitende Globalisierung des Terrors“ (S. 578) anspielt, ist übrigens der Titel eines Buches des Konservativen Winfried Martini, der vor über einem halben Jahrhundert ein „Kritik des Westens“ verfasst hatte. ${ }^{5}$ Im Gegensatz zu Martini stellt das Kapitel aber nicht auf die innere, sondern auf die äußere Bedrohung ab.

Anfangs zeichnet Winkler den großen Globalisierungsschub in der gleichsam unipolaren Welt nach. Der Kosovokrieg, geführt ohne ein Mandat des Sicherheitsrates der Vereinten Nationen, löste zumal in Deutschland heftige Kontroversen aus, die ihre Argumentationsmuster aus der Vergangenheit bezogen. Der Parole „Nie wieder Krieg“ stand das von Joschka Fischer verfochtene Diktum „Nie wieder Auschwitz“ gegenüber. Ausgerechnet die rotgrüne Regierung unterstützte 1999 den von Winkler goutierten ersten Einsatz deutscher Soldaten im Ausland. Nach dem Anschlag vom 11. September 2001 und dem „Krieg gegen

4 Vgl. Barbara Tuchman, In Geschichte denken. Essays, Düsseldorf 1982, S. 37.

5 Vgl. Winfried Martini, Das Ende aller Sicherheit. Eine Kritik des Westens, Stuttgart 1954. 
den Terror" änderte sich das Verhältnis zwischen den Partnern des Westens. Der US-amerikanische Bellizismus gefiel nicht allen europäischen Demokratien, die wiederum mit der europäischen Einigung nicht recht vorankamen. So scheiterte nach dem französischen „Non“ und dem niederländischen „Nee“ 2005 der Europäische Verfassungsvertrag. Zu Recht spricht der Autor von einem „Elitenprojekt“ (S. 269). Winkler, in der Vergangenheit mehrfach scharf gegen einen Beitritt der Türkei in die EU argumentierend ${ }^{6}$, urteilt jetzt verhältnismäßig milde und plädiert - wie bereits früher ${ }^{7}$ - für eine „privilegierte Partnerschaft" (S. 285). Im Vergleich zu den internationalen Konflikten geraten die Reformen und Kontroversen in den einzelnen Staaten etwas in den Hintergrund.

Die Proportionen zwischen den drei Großkapiteln stimmen nicht recht. Auf gut 300 Seiten fängt der Autor die Jahre von 1991 bis 2008 ein, auf über 250 Seiten die Zeit von 2008 bis 2014, darunter auf 100 Seiten vor allem die Jahre 2013/14. Wegen dieser etwas kurzatmig-aktualistischen Sichtweise wird die Ost-West-Konfrontation um die Ukraine allein auf fast 40 Seiten ausgebreitet. Voller Verve zieht Winkler gegen den „Putinismus“ zu Felde, dem es an einer „geschlossenen Weltanschauung“ (S. 578) fehle. Kurz nach Abschluss seines Werkes unterzeichneten über 60 Persönlichkeiten aus unterschiedlichen politischen Lagern (unter anderem Herta Däubler-Gmelin, Erhard Eppler, Roman Herzog, Burkhard Hirsch, Margot Käßmann, Lothar de Maizière, Otto Schily, Gerhard Schröder) einen von Walther Stützle (SPD), Horst Teltschik (CDU) und Antje Vollmer (Grüne) initiierten Aufruf an die Bundesregierung, die Bundestagsabgeordneten und die Medien, der gewisses Verständnis in puncto russischer Ukraine-Politik durchklingen ließ. ${ }^{8}$ Der Autor bezieht in einem „Spiegel“-Interview heftig dagegen Stellung: „Ich frage mich, ob den Unterzeichnern das Bewusstsein fehlt, dass sie dabei sind, die EU zu spalten und die NATO dazu."9

Winklers „Rückblick und Ausblick“ ist gleichsam eine konzise Zusammenfassung der vier Bände: „Vom normativen Projekt zum normativen Prozess“. Hier greift er die Idee des Westens auf, lässt seine Position Revue passieren, läuft noch einmal zu großer Form auf. Der Dualismus von geistlicher und weltlicher Herrschaft im Mittelalter habe den Weg zu Pluralismus und Individualismus geebnet. Aus der Amerikanischen und der Französischen Revolution sei das Projekt des Westens erwachsen. „Das normative Projekt des Westens wurde zum normativen Prozess, weil es von Anfang an immer auch als Korrektiv zur jeweiligen politischen und gesellschaftlichen Praxis wirkte“ (S. 587). Das Ende des Ersten Weltkrieges war ebenso eine Epochenwende wie das Ende des Zweiten, die Machtübernahme Hitlers ebenso wie die Gorbatschows, allerdings in anderer Hinsicht. Das Projekt des Westens ist gefährdet (durch Auswüchse des Westens selbst) wie gefährlich (für seine Gegner): „Die Wühlarbeit des normativen Projekts des Westens aber, der Ideen der unveräußerlichen Menschenrechte, der Herrschaft des Rechts, der Gewaltenteilung, der Volkssouveränität und der repräsentativen Demokratie ist noch lange nicht zu Ende“ (S. 611). Diese etwas sibyllinischen Sätze, mit denen das Werk endet, bedeuten wohl dreierlei: Zum ersten ist die

6 Vgl. Heinrich August Winkler, Grenzen der Erweiterung. Die Türkei ist kein Teil des Projekts Europa, in: Internationale Politik, 58. Jg. (2003), H. 2, S. 59 - 66.

7 Vgl. ders., Wir erweitern uns zu Tode, in: Die Zeit vom 7. November 2002, S. 6.

8 Vgl. „Wieder Krieg in Europa? Nicht in unserem Namen!“, in: Die Zeit online vom 5. Dezember 2014, http://www.zeit.de/politik/2014-12/aufruf-russland-dialog (Abruf am 2. Februar 2015).

9 Heinrich August Winkler, „Ein neuer Sonderweg“, in: Der Spiegel vom 29. Dezember 2014, S. 26 -29 , S. 28. 
Geschichte offen, zum zweiten das Projekt des Westens hartnäckig, zum dritten die Gegenwehr ebenso.

Winkler, Chronist und Analytiker der politischen Geschichte zugleich, präsentiert ein profundes Werk, das faktenreich informiert, argumentationsstark urteilt, Zusammenhänge entfaltet und glänzend formuliert, wobei die Steigerung des Wortes „extrem“ (S. 531, S. 594) irritiert. Die „Geschichte des Westens“ ist eine „Geschichte für den Westen“, ohne dass der Autor Apologie betreibt. Was das Einmannunternehmen Winkler in vier Bänden auf fast 5.000 Seiten geleistet hat, ist Herkulesarbeit. Wer jeden Tag fast drei Seiten zu Papier bringt (nur bezogen auf die "Geschichte des Westens“), benötigt langen Atem. Der Respekt des Lesers gehört dem Berliner Historiker, der, im achten Lebensjahrzehnt stehend, innerhalb von sechs Jahren ein solch monumentales Werk vorgelegt hat, selbst wenn Neunmalkluge den einen oder anderen - etwa ideengeschichtlichen - Aspekt missen mögen. Ob diese meisterhafte Geschichte je überboten wird?

Eckhard Jesse

\section{Bilanz der Föderalismusreform: umfassende Dokumentation mit analytischen Defiziten}

Schneider, Hans-Peter: Der neue deutsche Bundesstaat. Bericht über die Umsetzung der Föderalismusreform I (Reihe: Föderalismus - Studien, Band 25), Nomos Verlagsgesellschaft, BadenBaden 2013, 829 Seiten, $€ 189$,-.

Am 1. September 2006 traten im Rahmen der ersten Stufe der Föderalismusreform 25 Änderungen beziehungsweise Ergänzungen von Grundgesetzartikeln in Kraft. Die Einschätzung der Resultate ist in Politik und Wissenschaft noch immer umstritten. Zwar sind zu dieser Fragestellung eine Reihe von wissenschaftlichen Publikationen erschienen, was bis dato aber fehlte, waren Studien zur Umsetzung der Verfassungsreform. Der vorliegende Band unternimmt es, dieses Forschungsdesiderat zu schließen.

Auf über 800 Seiten legt der Verfassungsrechtler und Föderalismusforscher Hans-Peter Schneider den Abschlussbericht seines Projekts zur Analyse der umfangreichsten Teilrevision der bundesdeutschen Verfassung vor. Das Forschungsvorhaben wurde von 2008 bis 2012 unter der wissenschaftlichen Mitarbeit von Jutta Kramer und Till Bender am „Deutschen Institut für Föderalismusforschung e.V.“ durchgeführt. Die Forschungsfrage lautete, ob und in welchem Maße die Ziele der Föderalismusreform I erfolgreich umgesetzt wurden und wo weiterer Handlungsbedarf für Änderungen lokalisiert werden könne (S. 24 f.). Methodisch beruht die Analyse auf der Auswertung von über 1.200 schriftlichen Dokumenten. Für das Projekt wurde eigens eine Datenbank aufgebaut. Leider liegt der Publikation keine CD-ROM bei, die einen Zugang zum Datenmaterial ermöglicht.

Der vorgelegte Abschlussbericht ist als Implementations- und Evaluationsstudie angelegt (S. 6). Entsprechend gliedert sich der Band in zwei unterschiedliche Teilbereiche. Der erste sehr umfangreiche - Darstellung und Bestandsaufnahme - hat einen beschreibend dokumentierenden Charakter. Bewusst verzichtet der Autor auf die systematische Einbeziehung der vielgestaltigen wissenschaftlichen Literatur. Den Kern bildet die detaillierte und ausführliche Darstellung der Veränderungen des Grundgesetzes. Der zweite Teil - Auswertung und 